\title{
On Hilfer generalized proportional fractional derivative
}

Idris Ahmed ${ }^{1,3}$, Poom Kumam $^{1,2^{*}}$ (D), Fahd Jarad ${ }^{4}$, Piyachat Borisut ${ }^{1}$ and Wachirapong Jirakitpuwapat ${ }^{1}$

\section{"Correspondence:}

poom.kum@kmutt.ac.th

${ }^{1}$ KMUTTFixed Point Research Laboratory, KMUTT-Fixed Point

Theory and Applications Research Group (KMUTT-FPTA), Department

of Mathematics, Room SCL 802 Fixed Point Laboratory, Science Laboratory Building, Faculty of Science, King Mongkut's University of Technology Thonburi (KMUTT), 126 Pracha-Uthit Road, Bang Mod, Thrung Khru, Bangkok 10140,

Thailand

2Department of Medical Research, China Medical University Hospital, China Medical University, Taichung 40402, Taiwan

Full list of author information is available at the end of the article

\begin{abstract}
Motivated by the Hilfer and the Hilfer-Katugampola fractional derivative, we introduce in this paper a new Hilfer generalized proportional fractional derivative, which unifies the Riemann-Liouville and Caputo generalized proportional fractional derivative. Some important properties of the proposed derivative are presented. Based on the proposed derivative, we consider a nonlinear fractional differential equation with nonlocal initial condition and show that this equation is equivalent to the Volterra integral equation. In addition, the existence and uniqueness of solutions are proven using fixed point theorems. Furthermore, we offer two examples to clarify the results.
\end{abstract}

MSC: 26A33; 34A12; 34A43; 34D20

Keywords: Existence; Proportional fractional derivative; Fixed point theorems; Nonlocal condition; Volterra integral equation

\section{Introduction}

Fractional calculus has been concerned with integrals and derivatives of arbitrary noninteger order of functions. In recent years, several researchers in the field of fractional calculus have brought attention to the search for the best fractional derivative, which will be used to model real world problems. Fractional calculus is as old as the classical calculus whose equations are often considered unable to model certain complex systems and it turned out that the methods used in the fractional calculus are splendid when modeling long-memory processes and many phenomena that occur in physics, chemistry, electricity, mechanics and many other disciplines $[10,13,17,21,31,32,34,35,38-40]$.

However, scientists felt the need for other types of fractional operators restricted to Riemann-Liouville fractional operators and Caputo fractional derivatives until the turn of this century. Many scientists proposed a variety of new fractional operators which contributed to the growth of the field of fractional calculus [9, 14, 15, 24, 25, 27-29, 37]. It is worth noting that the fractional operators proposed in this work are unique instances of fractional integrals/derivatives in relation to another function described in $[4,5,22,41]$. But all of these operators possess one of the most important peculiarities of fractional operators: nonlocality.

(c) The Author(s) 2020. This article is licensed under a Creative Commons Attribution 4.0 International License, which permits use sharing, adaptation, distribution and reproduction in any medium or format, as long as you give appropriate credit to the original author(s) and the source, provide a link to the Creative Commons licence, and indicate if changes were made. The images or other third party material in this article are included in the article's Creative Commons licence, unless indicated otherwise in a credit line to the material. If material is not included in the article's Creative Commons licence and your intended use is not permitted by statutory regulation or exceeds the permitted use, you will need to obtain permission directly from the copyright holder. To view a copy of this licence, visit http://creativecommons.org/licenses/by/4.0/ 
In [30], the authors introduced a local derivative with a non-integer order and called it a conformable derivative. The process of conceptualization of these local derivatives will lead to the rediscovery of the nonlocal fractional operators described in [28, 29]. We explain the key principles of the conformable derivative and suggest a derivative consistent with the left and right versions. Once again, the nonlocal fractional version as proposed in $[1,12]$ is found in [27].

In all types of fractional calculus or calculus with derivative, the order zero for a function should be equal to the function. The conformable derivative lacks this essential property of every derivative and in fact it is a deficit. In order to circumvent this deficit, the authors in $[6,7]$ redefined the conformal derivative to yield the function itself when this local derivative is of the order of zero. Following this was work by Jarad et al. [23] where the authors suggested the fractional version of the redefined conformable derivative. The existence and uniqueness of solutions belong to the most important qualitative properties of fractional differential equations. The existence and uniqueness of solutions to fractional differential equations that include different types of fractional derivatives and initial/boundary conditions were tackled by several mathematicians (see [2, 3, 8, 11, 16, 19, 20, 26, 42-45] and the references cited therein).

Motivated by [18, 36], we propose a new fractional derivative (simply known as Hilfer generalized proportional fractional derivative). Therefore, in the context of the defined derivative, we discuss the existence and uniqueness of solutions for a certain type of nonlinear fractional differential equations with nonlocal initial condition. The Hilfer generalized proportional fractional differential equation is of the following form:

$$
\left\{\begin{array}{l}
\mathcal{D}_{a^{+}}^{p, q, \rho} x(t)=f(t, x(t)), \quad t \in J=[a, T], T>a \geq 0, \\
\mathcal{I}_{a^{+}}^{1-\gamma, \rho} x(a)=\sum_{i=1}^{m} c_{i} x\left(\tau_{i}\right), \quad p \leq \gamma=p+q-p q, \tau_{i} \in(a, T),
\end{array}\right.
$$

where $\mathcal{D}_{a^{+}}^{p, q, \rho}(\cdot)$ is the Hilfer generalized proportional fractional derivative of order $(0<p<$ 1), $\mathcal{I}_{a^{+}}^{1-\gamma, \rho}(\cdot)$ is the generalized proportional fractional integral of order $1-\gamma>0, c_{i} \in \mathbb{R}$, $f: J \times \mathbb{R} \rightarrow \mathbb{R}$ is a continuous function and $\tau_{i} \in J$ satisfying $a<\tau_{1}<\cdots<\tau_{m}<T$ for $i=$ $1, \ldots, m$. To the best of our knowledge no one has discussed the existence and uniqueness of solutions of (1.1).

The rest of the paper is structured as follows. In Sect. 2, we shall review some basic definitions and theoretical results that we need to proceed. We describe our proposed derivatives in Sect. 3, the Hilfer generalized proportional derivatives along with some of the preliminary properties. In addition, we also investigate the comparability between an initial value problem and an integral equation of Volterra, from which we prove the existence and uniqueness of the solution using fixed point theorems of Banach and Kransnoselskii's. Moreover, two examples were given to clarify the results. The conclusion of the paper is given in Sect. 4.

\section{Preliminaries and theoretical results}

We offer some preliminary details, results and definitions of fractional calculus in this section, which are important throughout this paper. 
Let $-\infty<a<b<\infty$ be finite and infinite intervals on $\mathbb{R}_{+}$. Denote by $\mathcal{C}[a, b]$, the spaces of the continuous function $f$ on $[a, b]$ with norm defined by [32]

$$
\|f\|_{\mathcal{C}[a, b]}=\max _{t \in[a, b]}|f(t)|
$$

and $\mathcal{A C}^{n}[a, b]$, the space of $n$ times absolutely continuous differentiable functions, given by

$$
\mathcal{A C}^{n}[a, b]=\left\{f:(a, b] \rightarrow \mathbb{R} ; f^{n-1} \in \mathcal{A C}([a, b])\right\} .
$$

The weighted space $\mathcal{C}_{\gamma}[a, b]$ of a functions $f$ on $(a, b]$ is defined by

$$
\mathcal{C}_{\gamma}[a, b]=\left\{f:(a, b] \rightarrow \mathbb{R} ;(t-a)^{\gamma} f(t) \in \mathcal{C}([a, b])\right\}, \quad 0 \leq \gamma<1,
$$

with the norm

$$
\|f\|_{\mathcal{C}_{\gamma}[a, b]}=\left\|(t-a)^{\gamma} f(t)\right\|_{\mathcal{C}[a, b]}=\max _{t \in[a, b]}\left|(t-a)^{\gamma} f(t)\right|
$$

The weighted space $\mathcal{C}_{\gamma}^{n}[a, b]$ of the functions $f$ on $(a, b]$ is defined by

$$
\mathcal{C}_{\gamma}^{n}[a, b]=\left\{f:(a, b] \rightarrow \mathbb{R} ; f(t) \in \mathcal{C}^{n-1}([a, b]) ; f^{n}(t) \in \mathcal{C}_{\gamma}([a, b])\right\}, \quad 0 \leq \gamma<1,
$$

with the norm

$$
\|f\|_{\mathcal{C}_{\gamma}^{n}[a, b]}=\sum_{k=0}^{n-1}\left\|f^{k}\right\|_{\mathcal{C}[a, b]}+\left\|f^{n}\right\|_{\mathcal{C}_{\gamma}[a, b]} .
$$

Clearly, $\mathcal{C}_{\gamma}^{0}[a, b]=\mathcal{C}_{\gamma}[a, b]$, if $n=0$.

Definition 2.1 ([32]) Suppose $f \in L^{1}([a, b], \mathbb{R})$. Then the fractional operator

$$
I_{a^{+}}^{p} f(t)=\frac{1}{\Gamma(p)} \int_{a}^{t}(t-\mu)^{p-1} f(\mu) d \mu, \quad p>0, t>a, n \in \mathbb{N},
$$

is referred to as the Riemann-Liouville integral of order $p$ with the lower limit $a^{+}$of the function $f$, where $\Gamma(\cdot)$ denotes the classical gamma function.

Definition 2.2 ([32]) Let $f \in \mathcal{C}([a, b])$. Then the fractional operator

$$
{ }^{L} D_{a+}^{p} f(t)=\frac{1}{\Gamma(n-p)} \frac{d^{n}}{d t^{n}} \int_{a}^{t}(t-\mu)^{n-p-1} f(\mu) d \mu, \quad p>0, t>a, n-1<p<n, n \in \mathbb{N},
$$

is called the Riemann-Liouville fractional derivative of order $p$ with the lower limit $a^{+}$of the function $f$, where $\Gamma(\cdot)$ denotes the gamma function.

Definition 2.3 ([32]) Let $f \in \mathcal{C}^{n}([a, b])$. Then the fractional operator

$$
{ }^{C} D_{a+}^{p} f(t)=\frac{1}{\Gamma(n-p)} \int_{a}^{t}(t-\mu)^{n-p-1} f^{n}(\mu) d \mu, \quad p>0, n-1<p<n, n \in \mathbb{N},
$$


is referred to the Caputo fractional derivative of order $p$ with the lower limit $a^{+}$of the function $f$.

Definition 2.4 ([23]) If $\rho \in(0,1]$ and $p \in \mathbb{C}, \operatorname{Re}(p)>0$. Then the fractional operator

$$
I_{a^{+}}^{p, \rho} f(t)=\frac{1}{\rho^{p} \Gamma(p)} \int_{a}^{t} e^{\frac{\rho-1}{\rho}(t-\mu)}(t-\mu)^{p-1} f(\mu) d \mu, \quad t>a,
$$

is called the left-sided generalized proportional integral of order $p$ of the function $f$.

Definition 2.5 ([23]) The left generalized proportional fractional derivative of order $p$ and $\rho \in(0,1]$ of a function $f$ is defined by

$$
D_{a^{+}}^{p, \rho} f(t)=\frac{D^{n, \rho}}{\rho^{n-p} \Gamma(n-p)} \int_{a}^{t} e^{\frac{\rho-1}{\rho}(t-\mu)}(t-\mu)^{n-p-1} f(\mu) d \mu, \quad p \in \mathbb{C}, \operatorname{Re}(p)>0,
$$

where $\Gamma(\cdot)$ is the Gamma function and $n=[p]+1$.

Definition 2.6 ([23]) Let $\rho \in(0,1]$. Then the fractional operator

$$
\begin{aligned}
{ }^{C} D_{a^{+}}^{p, \rho} f(t)= & \frac{1}{\rho^{n-p} \Gamma(n-p)} \\
& \times \int_{a}^{t} e^{\frac{\rho-1}{\rho}(t-\mu)}(t-\mu)^{n-p-1}\left(D^{n, \rho} f\right)(\mu) d \mu, \quad p \in \mathbb{C}, \operatorname{Re}(p)>0,
\end{aligned}
$$

is referred to as the left-sided generalized proportional fractional derivative in the sense of Caputo of order $p$ of the function $f$, where $\Gamma(\cdot)$ is the gamma function and $n=[p]+1$.

Remark 2.7 Note that if $\rho=1$ Definitions 2.4, 2.5 and 2.6 coincide with the classical definitions of the Riemann-Liouville fractional integral, the Riemann-Liouville fractional derivative and the Caputo fractional derivative (see Definitions 2.1, 2.2 and 2.3).

The following are certain important properties of the generalized proportional fractional integral and derivative.

Proposition $2.8([23])$ Let $p, \delta \in \mathbb{C}$ such that $\operatorname{Re}(p) \geq 0$ and $\operatorname{Re}(\delta)>0$. Then for any $\rho \in$ $(0,1]$ we have

$$
\begin{aligned}
& \left(I_{a^{+}}^{p, \rho} e^{\frac{\rho-1}{\rho} s}(s-a)^{\delta-1}\right)(t)=\frac{\Gamma(\delta)}{\rho^{p} \Gamma(\delta+p)} e^{\frac{\rho-1}{\rho} t}(t-a)^{\delta+p-1} \\
& \left(D_{a^{+}}^{p, \rho} e^{\frac{\rho-1}{\rho} s}(s-a)^{\delta-1}\right)(t)=\frac{\rho^{p} \Gamma(\delta)}{\Gamma(\delta-p)} e^{\frac{\rho-1}{\rho} t}(t-a)^{\delta-p-1} \\
& \left(I_{b}^{p, \rho} e^{\frac{\rho-1}{\rho}(b-s)}(b-s)^{\delta-1}\right)(t)=\frac{\Gamma(\delta)}{\rho^{p} \Gamma(\delta+p)} e^{\frac{\rho-1}{\rho}(b-t)}(b-t)^{\delta+p-1}, \\
& \left(D_{b}^{p, \rho} e^{\frac{\rho-1}{\rho}(b-s)}(b-s)^{q-1}\right)(t)=\frac{\rho^{p} \Gamma(\delta)}{\Gamma(\delta-p)} e^{\frac{\rho-1}{\rho}(b-t)}(b-t)^{\delta-p-1} .
\end{aligned}
$$

Theorem 2.9 ([23]) Let $\rho \in(0,1], \operatorname{Re}(p)>0$ and $\operatorname{Re}(q)>0$. If $f \in \mathcal{C}([a, b], \mathbf{R})$, then

$$
I_{a^{+}}^{p, \rho}\left(I_{a^{+}}^{q, \rho} f\right)(t)=I_{a^{+}}^{q, \rho}\left(I_{a^{+}}^{p, \rho} f\right)(t)=\left(I_{a^{+}}^{p+q, \rho} f\right)(t), \quad t \geq a .
$$


Theorem 2.10 ([23]) Suppose $\rho \in(0,1]$ and $0 \leq m<[\operatorname{Re}(p)]+1$. If $f \in L^{1}([a, b])$. Then

$$
D_{a^{+}}^{m, \rho}\left(I_{a^{+}}^{p, \rho} f\right)(t)=\left(I_{a^{+}}^{p-m, \rho} f\right)(t), \quad t>a .
$$

Corollary 2.11 ([23]) If $0<\operatorname{Re}(q)<\operatorname{Re}(p)$ and $m-1<\operatorname{Re}(q) \leq m$. Then we get

$$
D_{a^{+}}^{q, \rho} I_{a^{+}}^{p, \rho} f(t)=I_{a^{+}}^{p-q, \rho} f(t) .
$$

Theorem 2.12 ([23]) Let $f \in L^{1}([a, b]), \operatorname{Re}(p)>0$ and $\rho \in(0,1]$. Then

$$
D_{a^{+}}^{p, \rho} I_{a^{+}}^{p, \rho} f(t)=f(t), \quad t \geq a, n=[\operatorname{Re}(p)]+1 .
$$

Lemma 2.13 ([23]) If $p>0, \rho \in(0,1]$ and $m \in \mathbb{Z}_{+}$. Then

$$
\left(I_{a^{+}}^{p, \rho} D_{a^{+}}^{m, \rho} f\right)(t)=\left(D_{a^{+}}^{m, \rho} I_{a^{+}}^{p, \rho} f\right)(t)-\sum_{k=0}^{m-1} \frac{e^{\frac{\rho-1}{\rho}(t-a)}(t-a)^{p-m+k}}{\rho^{p-m+k} \Gamma(p+k-m+1)}\left(D_{a^{+}}^{k, \rho} f\right)(a) .
$$

In particular, if $m=1$, we obtain

$$
\left(I_{a^{+}}^{p, \rho} D_{a^{+}}^{\rho} f\right)(t)=\left(D_{a^{+}}^{\rho} I_{a^{+}}^{p, \rho} f\right)(t)-\frac{e^{\frac{\rho-1}{\rho}(t-a)}(t-a)^{p-1}}{\rho^{p-1} \Gamma(p)} f(a) .
$$

Theorem 2.14 ([23]) Let $\operatorname{Re}(p)>0, n=-[-\operatorname{Re}(p)], f \in L_{1}(a, b)$ and $\left(I_{a^{+}}^{p, \rho} f\right)(t) \in A C^{n}[a, b]$. Then

$$
\left(I_{a^{+}}^{p, \rho} D_{a^{+}}^{p, \rho} f\right)(t)=f(t)-e^{\frac{\rho-1}{\rho}(t-a)} \sum_{j=1}^{n} \frac{(t-a)^{p-j}}{\rho^{p-j} \Gamma(p-j+1)}\left(I_{a}^{j-p, \rho} f\right)\left(a^{+}\right) .
$$

\section{Main results}

We introduce the Hilfer generalized proportional fractional derivative in this section and discuss some of its properties. Additionally, we demonstrate the equivalence between the proposed problem (1.1) and the integral equation of Volterra type. In addition, we prove the existence and uniqueness of solutions of problem (1.1) by employing the fixed point theorems.

Definition 3.1 Let $n-1<p<n, \rho \in(0,1]$ and $0 \leq q \leq 1$, with $n \in \mathbb{N}$. The left-sided/rightsided Hilfer generalized proportional fractional derivative of order $p$ and type $q$ of a function $f$ is defined by

$$
\left(\mathcal{D}_{a^{ \pm}}^{p, q, \rho} f\right)(x)=\mathcal{I}_{a^{ \pm}}^{q(n-p), \rho}\left[D^{\rho}\left(\mathcal{I}_{a^{ \pm}}^{(1-q)(n-p), \rho} f\right)\right](x),
$$

where $D^{\rho} f(x)=(1-\rho) f(x)+\rho f^{\prime}(x)$ and $\mathcal{I}$ is the generalized proportional fractional integral defined in Eq. (2.1).

In particular, if $n=1$, Definition 3.1 is equivalent with

$$
\left(\mathcal{D}_{a^{ \pm}}^{p, q, \rho} f\right)(x)=\mathcal{I}_{a^{ \pm}}^{q(1-p), \rho}\left[D^{\rho}\left(\mathcal{I}_{a^{ \pm}}^{(1-q)(1-p), \rho} f\right)\right](x) .
$$


Thus, throughout this paper, we discuss the case where $n=1,0<p<1,0 \leq q \leq 1$ and $\gamma=p+q-p q$.

Remark 3.2 It is worthwhile to specify that:

- The derivative is used as an interpolator between the Riemann-Liouville and Caputo generalized proportional fractional derivative, respectively, since

$$
\mathcal{D}_{a^{ \pm}}^{p, q, \rho} f= \begin{cases}D^{\rho} \mathcal{I}_{a^{ \pm}}^{(1-p), \rho} f, & q=0(\text { see Definition 2.5), } \\ \mathcal{I}_{a^{ \pm}}^{(1-p), \rho} D^{\rho} f, & q=1 \text { (see Definition 2.6) }\end{cases}
$$

- The parameter $\gamma$ satisfies

$$
0<\gamma \leq 1, \quad \gamma \geq p, \quad \gamma>q, \quad 1-\gamma<1-q(1-p) .
$$

Property 3.3 The operator $\mathcal{D}_{a^{+}}^{p, q, \rho}$ can be simplified as

$$
\mathcal{D}_{a^{+}}^{p, q, \rho} f=\mathcal{I}_{a^{+}}^{q(1-p), \rho} D^{\rho} \mathcal{I}_{a^{+}}^{(1-\gamma), \rho} f=\mathcal{I}_{a^{+}}^{q(1-p), \rho} \mathcal{D}_{a^{+}}^{\gamma, \rho} f, \quad \gamma=p+q-p q .
$$

Proof In view of Equation (3.2) and Definition 2.5,

$$
\begin{aligned}
\left(\mathcal{D}_{a^{+}}^{p, q, \rho} f\right)(x) & =\mathcal{I}_{a^{+}}^{q(1-p), \rho}\left[\mathcal{D}^{\rho}\left(\mathcal{I}_{a^{+}}^{(1-q)(1-p), \rho} f\right)\right](x) \\
& =\mathcal{I}_{a^{+}}^{q(1-p), \rho}\left\{\frac{D^{\rho}}{\rho^{(1-\gamma)} \Gamma((1-\gamma))} \int_{a}^{t} e^{\frac{\rho-1}{\rho}(t-\tau)}(t-\tau)^{(1-\gamma)-1} f(\tau) d \tau\right\} \\
& =\left(\mathcal{I}_{a^{+}}^{q(1-p), \rho} \mathcal{D}^{\gamma, \rho} f\right)(x) .
\end{aligned}
$$

We consider the following weighted spaces of continuous function on $(a, b]$ :

$$
\mathcal{C}_{1-\gamma}^{p, q}[a, b]=\left\{f \in \mathcal{C}_{1-\gamma}[a, b], \mathcal{D}_{a^{+}}^{p, q, \rho} f \in \mathcal{C}_{1-\gamma}[a, b]\right\}
$$

and

$$
\mathcal{C}_{1-\gamma}^{\gamma}[a, b]=\left\{f \in \mathcal{C}_{1-\gamma}[a, b], \mathcal{D}_{a^{+}}^{\gamma, \rho} f \in \mathcal{C}_{1-\gamma}[a, b]\right\} .
$$

Since $\mathcal{D}_{a^{+}}^{p, q, \rho}=\mathcal{I}_{a^{+}}^{q(1-p), \rho} \mathcal{D}_{a^{+}}^{\gamma, \rho}$,

$$
\mathcal{C}_{1-\gamma}^{\gamma}[a, b] \subset \mathcal{C}_{1-\gamma}^{p, q}[a, b]
$$

Lemma 3.4 Suppose $0<p<1, \rho \in(0,1]$ and $0 \leq \gamma<1$. Iff $\in \mathcal{C}_{\gamma}[a, b]$ then

$$
\mathcal{I}_{a^{+}}^{p, \rho} f(a)=\lim _{x \rightarrow a^{+}} \mathcal{I}_{a^{+}}^{p, \rho} f(x)=0, \quad 0 \leq \gamma<p .
$$

Proof Considering $f \in \mathcal{C}[a, b]$, it implies that $f \in \mathcal{C}_{\gamma}[a, b]$ and $(x-a)^{\gamma} \in \mathcal{C}[a, b]$. Therefore, there exists $M>0$ for which

$$
(x-a)^{\gamma} f(x)<M, \quad \text { for all } x \in[a, b],
$$


and

$$
\left|\mathcal{I}_{a^{+}}^{p, \rho} e^{\frac{\rho-1}{\rho} x} f(x)\right|<M\left[\mathcal{I}_{a^{+}}^{p, \rho} e^{\frac{\rho-1}{\rho} t}(t-a)^{-\gamma}\right](x) .
$$

It follows from Proposition 2.8, that

$$
\left|\mathcal{I}_{a^{+}}^{p, \rho} e^{\frac{\rho-1}{\rho} x} f(x)\right|<M\left[\frac{\Gamma(1-\gamma)}{\Gamma(p+1-\gamma)} e^{\frac{\rho-1}{\rho} x}(x-a)^{p-\gamma}\right],
$$

which implies that the right-hand side $\rightarrow 0$ as $x \rightarrow a^{+}$.

Lemma 3.5 Let $0<p<1, \rho \in(0,1], 0 \leq q \leq 1$ and $\gamma=p+q-p q$. If $\in \mathcal{C}_{1-\gamma}^{\gamma}[a, b]$ then

$$
\mathcal{I}_{a^{+}}^{\gamma, \rho} \mathcal{D}_{a^{+}}^{\gamma, \rho} f=\mathcal{I}_{a^{+}}^{p, \rho} \mathcal{D}_{a^{+}}^{p, q, \rho} f
$$

and

$$
\mathcal{D}_{a^{+}}^{\gamma, \rho} \mathcal{I}_{a^{+}}^{p, \rho} f=\mathcal{D}_{a^{+}}^{q(1-p), \rho} f
$$

Proof Making use of Theorem 2.9 and Property 3.3,

$$
\begin{aligned}
\mathcal{I}_{a^{+}}^{\gamma, \rho} \mathcal{D}_{a^{+}}^{\gamma, \rho} f & =\mathcal{I}_{a^{+}}^{\gamma, \rho}\left(\mathcal{I}_{a^{+}}^{-q(1-p), \rho} \mathcal{D}_{a^{+}}^{p, q, \rho} f\right) \\
& =\mathcal{I}_{a^{+}}^{p+q-p q, \rho} \mathcal{I}_{a^{+}}^{-q(1-p), \rho} \mathcal{D}_{a^{+}}^{p, q, \rho} f \\
& =\mathcal{I}_{a^{+}}^{p, \rho} \mathcal{D}_{a^{+}}^{p, q, \rho} f
\end{aligned}
$$

Furthermore, in view of Theorem 2.9 and Eq. (3.2), we can see that

$$
\begin{aligned}
\mathcal{D}_{a^{+}}^{\gamma, \rho} \mathcal{I}_{a^{+}}^{p, \rho} f & =\mathcal{D}_{a^{+}}^{\rho} \mathcal{I}_{a^{+}}^{1-\gamma, \rho} \mathcal{I}_{a^{+}}^{p, \rho} f \\
& =\mathcal{D}_{a^{+}}^{\rho} \mathcal{I}_{a^{+}}^{1-q+p q, \rho} f \\
& =\mathcal{D}_{a^{+}}^{q(1-p), \rho} f .
\end{aligned}
$$

Lemma 3.6 Suppose $f \in L^{1}(a, b)$ such that $\mathcal{D}_{a^{+}}^{q(1-p), \rho} f$ exists in $L^{1}(a, b)$. Then

$$
\mathcal{D}_{a^{+}}^{p, q, \rho} \mathcal{I}_{a^{+}}^{p, \rho} f=\mathcal{I}_{a^{+}}^{q(1-p), \rho} \mathcal{D}_{a^{+}}^{q(1-p), \rho} f .
$$

Proof It follows from Definition 2.5 and Eq. (3.2) that

$$
\begin{aligned}
\mathcal{D}_{a^{+}}^{p, q, \rho} \mathcal{I}_{a^{+}}^{p, \rho} f & =\mathcal{I}_{a^{+}}^{q(1-p), \rho} \mathcal{D}_{a^{+}}^{\rho} \mathcal{I}_{a^{+}}^{(1-q)(1-p), \rho} \\
& =\mathcal{I}_{a^{+}}^{q(1-p), \rho} \mathcal{D}_{a^{+}}^{\rho} \mathcal{I}_{a^{+}}^{1-q(1-p), \rho} \\
& =\mathcal{I}_{a^{+}}^{q(1-p), \rho} \mathcal{D}_{a^{+}}^{q(1-p), \rho} f
\end{aligned}
$$

Lemma 3.7 Let $0<p<1, \rho \in(0,1]$, and $0 \leq \gamma<1$. If $f \in \mathcal{C}_{\gamma}[a, b]$ and $\mathcal{I}_{a^{+}}^{1-p, \rho} f \in \mathcal{C}_{\gamma}^{1}[a, b]$, then

$$
\mathcal{I}_{a^{+}}^{p, \rho} \mathcal{D}_{a^{+}}^{p, \rho} f(x)=f(x)-e^{\frac{\rho-1}{\rho}(x-a)} \frac{(x-a)^{p-1}}{\rho^{p-1} \Gamma(p)}\left(I_{a}^{1-p, \rho} f\right)\left(a^{+}\right),
$$

for all $x \in(a, b]$. 
Proof The proof is similar to the ones in [23].

Lemma 3.8 Let $0<p<1, \rho \in(0,1], 0 \leq q \leq 1$ and $\gamma=p+q-p q$. If $f \in \mathcal{C}_{1-\gamma}[a, b]$ and $\mathcal{D}_{a^{+}}^{p, q, \rho} f$ then $\mathcal{D}_{a^{+}}^{p, q, \rho} \mathcal{I}_{a^{+}}^{p, \rho} f$ exists in $(a, b)$ and

$$
\mathcal{D}_{a^{+}}^{p, q, \rho} \mathcal{I}_{a^{+}}^{p, \rho} f(x)=f(x), \quad x \in(a, b]
$$

Proof Now, using Lemmas 3.4, 3.6 and 3.7, we have

$$
\begin{aligned}
\left(\mathcal{D}_{a^{+}}^{p, q, \rho} \mathcal{I}_{a^{+}}^{p, \rho} f\right)(x) & =\left(\mathcal{I}_{a^{+}}^{q(1-p), \rho} \mathcal{D}_{a^{+}}^{q(1-p), \rho} f\right)(x) \\
& =f(x)-e^{\frac{\rho-1}{\rho}(x-a)} \frac{(x-a)^{q(1-p)-1}}{\rho^{q(1-p)-1} \Gamma(q(1-p))}\left(I_{a}^{1-q(1-p), \rho} f\right)\left(a^{+}\right) \\
& =f(x) .
\end{aligned}
$$

Lemma 3.9 Let $0<p<1, \rho \in(0,1], 0 \leq q \leq 1$ and $0<\gamma<1$. If $\in \mathcal{C}_{1-\gamma}[a, b]$ and $\mathcal{I}_{a^{+}}^{1-\gamma, \rho} f$, then

$$
\mathcal{I}_{a^{+}}^{p, \rho} \mathcal{D}_{a^{+}}^{p, q, \rho} f(x)=f(x)-e^{\frac{\rho-1}{\rho}(x-a)} \frac{(x-a)^{\gamma-1}}{\rho^{\gamma-1} \Gamma(\gamma)}\left(I_{a}^{1-\gamma, \rho} f\right)\left(a^{+}\right), \quad x \in(a, b]
$$

Proof It follows from Definition 3.1 and Lemma 3.7 that

$$
\begin{aligned}
\left(\mathcal{I}_{a^{+}}^{p, \rho} \mathcal{D}_{a^{+}}^{p, q, \rho} f\right)(x) & =\mathcal{I}_{a^{+}}^{p, \rho}\left(\mathcal{I}_{a^{+}}^{\gamma-p, \rho} \mathcal{D}_{a^{+}}^{\gamma, \rho} f\right)(x) \\
& =\mathcal{I}_{a^{+}}^{\gamma, \rho} \mathcal{D}_{a^{+}}^{\gamma, \rho} f(x) \\
& =f(x)-e^{\frac{\rho-1}{\rho}(x-a)} \frac{(x-a)^{\gamma-1}}{\rho^{\gamma-1} \Gamma(\gamma)}\left(I_{a}^{1-\gamma, \rho} f\right)\left(a^{+}\right) .
\end{aligned}
$$

\subsection{Equivalent mixed-type Volterra integral equation}

The following lemma shows the equivalence between the proposed problem (1.1) and the Volterra integral equation.

Lemma 3.10 Let $0<p<1,0 \leq q \leq 1$ and $\gamma=p+q-p q$ and let $f: J \times \mathbb{R} \rightarrow \mathbb{R}$ be a function such that $f \in \mathcal{C}_{1-\gamma}[J, \mathbb{R}]$ for any $x \in \mathcal{C}_{1-\gamma}[J, \mathbb{R}]$. If $x \in \mathcal{C}_{1-\gamma}^{\gamma}[J, \mathbb{R}]$ then $x$ satisfies problem $(1.1)$ if and only if $x$ satisfies the mixed-type integral equation:

$$
\begin{aligned}
x(t)= & \frac{\Lambda}{\rho^{p} \Gamma(p)} e^{\frac{(\rho-1)}{\rho}(t-a)}(t-a)^{\gamma-1} \sum_{i=1}^{m} c_{i} \int_{a^{+}}^{\tau_{i}} e^{\frac{(\rho-1)}{\rho}\left(\tau_{i}-s\right)}\left(\tau_{i}-s\right)^{p-1} f(s, x(s)) d s \\
& +\frac{1}{\rho^{p} \Gamma(p)} \int_{a^{+}}^{t} e^{\frac{(\rho-1)}{\rho}(t-s)}(t-s)^{p-1} f(s, x(s)) d s,
\end{aligned}
$$

where

$$
\Lambda=\frac{1}{\rho^{\gamma-1} \Gamma(\gamma)-\sum_{i=1}^{m} c_{i} e^{\frac{(\rho-1)}{\rho}\left(\tau_{i}-a\right)}\left(\tau_{i}-a\right)^{\gamma-1}} .
$$


Proof Suppose, $x \in \mathcal{C}_{1-\gamma}^{\gamma}[J, \mathbb{R}]$ be a solution of (1.1). We show that $x$ is also a solution of (3.4). In view of Lemma 3.9, we have

$$
\begin{aligned}
x(t)= & \frac{(t-a)^{\gamma-1}}{\rho^{\gamma-1} \Gamma(\gamma)} e^{\frac{(\rho-1)}{\rho}(t-a)} \mathcal{I}_{a^{+}}^{1-\gamma, \rho} x\left(a^{+}\right) \\
& +\frac{1}{\rho^{p} \Gamma(p)} \int_{a^{+}}^{t} e^{\frac{(\rho-1)}{\rho}(t-s)}(t-s)^{p-1} f(s, x(s)) d s .
\end{aligned}
$$

Now, substituting $t=\tau_{i}$ and multiplying both sides by $c_{i}$ in (3.6), we get

$$
c_{i} x\left(\tau_{i}\right)=\frac{\left(\tau_{i}-a\right)^{\gamma-1}}{\rho^{\gamma-1} \Gamma(\gamma)} e^{\frac{(\rho-1)}{\rho}\left(\tau_{i}-a\right)} c_{i} \mathcal{I}_{a^{+}}^{1-\gamma, \rho} x\left(a^{+}\right)+c_{i} \mathcal{I}_{a^{+}}^{p, \rho} f\left(\tau_{i}\right),
$$

which implies that

$$
\begin{aligned}
\sum_{i=1}^{m} c_{i} x\left(\tau_{i}\right)= & \frac{1}{\rho^{\gamma-1} \Gamma(\gamma)} \sum_{i=1}^{m} c_{i} e^{\frac{(\rho-1)}{\rho}\left(\tau_{i}-a\right)}\left(\tau_{i}-a\right)^{\gamma-1} \mathcal{I}_{a^{+}}^{1-\gamma, \rho} x\left(a^{+}\right) \\
& +\frac{1}{\rho^{p} \Gamma(p)} \sum_{i=1}^{m} c_{i} \int_{a^{+}}^{\tau_{i}} e^{\frac{(\rho-1)}{\rho}\left(\tau_{i}-s\right)}\left(\tau_{i}-s\right)^{p-1} f(s, x(s)) d s .
\end{aligned}
$$

From the initial condition $\mathcal{I}_{a^{+}}^{1-\gamma, \rho} x(a)=\sum_{i=1}^{m} c_{i} x\left(\tau_{i}\right)$, we get

$$
\mathcal{I}_{a^{+}}^{1-\gamma, \rho} x\left(a^{+}\right)=\frac{\rho^{\gamma-1} \Gamma(\gamma)}{\rho^{p} \Gamma(p)} \Lambda \sum_{i=1}^{m} c_{i} \int_{a^{+}}^{\tau_{i}} e^{\frac{(\rho-1)}{\rho}\left(\tau_{i}-s\right)}\left(\tau_{i}-s\right)^{p-1} f(s, x(s)) d s
$$

Therefore, the result follows by substituting (3.9) in (3.6). This shows that $x(t)$ satisfies (3.4).

Conversely, suppose that $x \in \mathcal{C}_{1-\gamma}^{\gamma}$ satisfies Eq. (3.4), then we show that $x$ also satisfies Eq. (1.1). Applying $\mathcal{D}_{a^{+}}^{\gamma, \rho}$ to both sides of (3.4) and in view of Proposition 2.8, Lemma 2.10 and Definition 3.1, we have

$$
\begin{aligned}
\mathcal{D}_{a^{+}}^{\gamma, \rho} & x(t) \\
= & \mathcal{D}_{a^{+}}^{\gamma, \rho}\left(\frac{\Lambda}{\rho^{p} \Gamma(p)} e^{\frac{(\rho-1)}{\rho}(t-a)}(t-a)^{\gamma-1} \sum_{i=1}^{m} c_{i} \int_{a^{+}}^{\tau_{i}} e^{\frac{(\rho-1)}{\rho}\left(\tau_{i}-s\right)}\left(\tau_{i}-s\right)^{p-1} f(s, x(s)) d s\right) \\
& +\mathcal{D}_{a^{+}}^{\gamma, \rho}\left(\frac{1}{\rho^{p} \Gamma(p)} \int_{a^{+}}^{t} e^{\frac{(\rho-1)}{\rho}(t-s)}(t-s)^{p-1} f(s, x(s)) d s\right) \\
= & \left(\mathcal{D}_{a^{+}}^{q(1-p), \rho} f(t, x(t))\right)(x) .
\end{aligned}
$$

Since $\mathcal{D}_{a^{+}}^{p, q, \rho} x \in \mathcal{C}_{1-\gamma}[J, \mathbb{R}]$, by the definition of $\mathcal{C}_{1-\gamma}^{\gamma}[J, \mathbb{R}]$ Eq. (3.10) implies that

$$
\mathcal{D}_{a^{+}}^{q(1-p), \rho} f=D^{\rho} \mathcal{I}_{a^{+}}^{1-q(1-\rho), \rho} f \in \mathcal{C}_{1-\gamma, \rho}[J, \mathbb{R}] .
$$

For $f \in \mathcal{C}_{1-\gamma}[J, \mathbb{R}]$ and from Lemma 2.12, we can see that $\mathcal{I}_{a^{+}}^{1-q(1-p), \rho} f \in \mathcal{C}_{1-\gamma, \rho}[, \mathbb{R}]$, this implies that $\mathcal{I}_{a^{+}}^{1-q(1-p), \rho} f \in \mathcal{C}_{1-\gamma}^{1}[, \mathbb{R}]$ from the definition of $\mathcal{C}_{\gamma}^{n}[, \mathbb{R}]$. 
Applying $\mathcal{I}_{a^{+}}^{q(1-p), \rho}$ on both sides of (3.10) and in view of Proposition 2.8, Lemma 3.7 and Definition 3.1,

$$
\begin{aligned}
\mathcal{I}_{a^{+}}^{q(1-p), \rho} \mathcal{D}_{a^{+}}^{\gamma, \rho} x(t) & =\mathcal{I}_{a^{+}}^{q(1-p), \rho} \mathcal{D}_{a^{+}}^{q(1-p), \rho} f(t, x(t)) . \\
& =f(t, x(t))-\frac{\left(\mathcal{I}_{a^{+}}^{1-q(1-p), \rho} f\right)(a)}{\Gamma(q(1-p))}(t-a)^{q(p-1)-1} \\
& =f(t, x(t)) .
\end{aligned}
$$

Hence, its remains to show that if $x \in \mathcal{C}_{1-\gamma}^{\gamma}[J, \mathbb{R}]$ satisfies (3.4), it also satisfies the initial condition. So, by applying $\mathcal{I}_{a^{+}}^{1-\gamma, \rho}$ to both sides of (3.4) and using Proposition 2.8, Theorem 2.9 and 2.11, we obtain

$$
\begin{aligned}
\mathcal{I}_{a^{+}}^{1-\gamma, \rho} x(t) & \\
= & \mathcal{I}_{a^{+}}^{1-\gamma, \rho}\left(\frac{\Lambda}{\rho^{p} \Gamma(p)} e^{\frac{(\rho-1)}{\rho}(t-a)}(t-a)^{\gamma-1} \sum_{i=1}^{m} c_{i} \int_{a^{+}}^{\tau_{i}} e^{\frac{(\rho-1)}{\rho}\left(\tau_{i}-s\right)}\left(\tau_{i}-s\right)^{p-1} f(s) d s\right) \\
& +\mathcal{I}_{a^{+}}^{1-\gamma ; \varphi}\left(\frac{1}{\rho^{p} \Gamma(p)} \int_{a^{+}}^{t} e^{\frac{(\rho-1)}{\rho}(t-s)}(t-s)^{p-1} f(s) d s\right) \\
= & \frac{\rho^{\gamma-1} \Gamma(\gamma)}{\rho^{p} \Gamma(p)} \Lambda e^{\frac{(\rho-1)}{\rho}(t-a)} \sum_{i=1}^{m} c_{i} \int_{a^{+}}^{\tau_{i}} e^{\frac{(\rho-1)}{\rho}\left(\tau_{i}-s\right)}\left(\tau_{i}-s\right)^{p-1} f(s) d s \\
& +\mathcal{I}_{a^{+}}^{1-q(1-p), \rho} f(t) .
\end{aligned}
$$

Taking the limit as $t \rightarrow a^{+}$in Eq. (3.12) and the fact that $1-q<1-p(1-r)$ give

$$
\mathcal{I}_{a^{+}}^{1-\gamma, \rho} x\left(a^{+}\right)=\frac{\rho^{\gamma-1} \Gamma(\gamma)}{\rho^{p} \Gamma(p)} \Lambda \sum_{i=1}^{m} c_{i} \int_{a^{+}}^{\tau_{i}} e^{\frac{(\rho-1)}{\rho}\left(\tau_{i}-s\right)}\left(\tau_{i}-s\right)^{p-1} f(s, x(s)) d s
$$

Substituting $t=\tau_{i}$ and multiplying through by $c_{i}$ in (3.4),

$$
\begin{aligned}
c_{i} x\left(\tau_{i}\right)= & \frac{\Lambda}{\rho^{p} \Gamma(p)} e^{\frac{(\rho-1)}{\rho}\left(\tau_{i}-a\right)}\left(\tau_{i}-a\right)^{\gamma-1} \sum_{i=1}^{m} c_{i} \int_{a^{+}}^{\tau_{i}} e^{\frac{(\rho-1)}{\rho}\left(\tau_{i}-s\right)}\left(\tau_{i}-s\right)^{p-1} f(s) d s \\
& +\frac{c_{i}}{\rho^{p} \Gamma(p)} \int_{a^{+}}^{\tau_{i}} e^{\frac{(\rho-1)}{\rho}\left(\tau_{i}-s\right)}\left(\tau_{i}-s\right)^{p-1} f(s, x(s)) d s,
\end{aligned}
$$

which implies that

$$
\begin{aligned}
\sum_{i=1}^{m} c_{i} x\left(\tau_{i}\right) & =\Lambda \sum_{i=1}^{m} c_{i} \mathcal{I}_{a^{+}}^{p, \rho} f\left(\tau_{i}\right) \sum_{i=1}^{m} c_{i} e^{\frac{(\rho-1)}{\rho}\left(\tau_{i}-a\right)}\left(\tau_{i}-a\right)^{\gamma-1}+\sum_{i=1}^{m} c_{i} \mathcal{I}_{a^{+}}^{p, \rho} f\left(\tau_{i}\right) \\
& =\sum_{i=1}^{m} c_{i} \mathcal{I}_{a^{+}}^{p, \rho} f\left(\tau_{i}\right)\left(1+\Lambda \sum_{i=1}^{m} c_{i} e^{\frac{(\rho-1)}{\rho}\left(\tau_{i}-a\right)}\left(\tau_{i}-a\right)^{\gamma-1}\right)
\end{aligned}
$$

Thus

$$
\sum_{i=1}^{m} c_{i} x\left(\tau_{i}\right)=\frac{\rho^{\gamma-1} \Gamma(\gamma)}{\rho^{p} \Gamma(p)} \Lambda \sum_{i=1}^{m} c_{i} \int_{a^{+}}^{\tau_{i}} e^{\frac{(\rho-1)}{\rho}\left(\tau_{i}-s\right)}\left(\tau_{i}-s\right)^{p-1} f(s, x(s)) d s .
$$




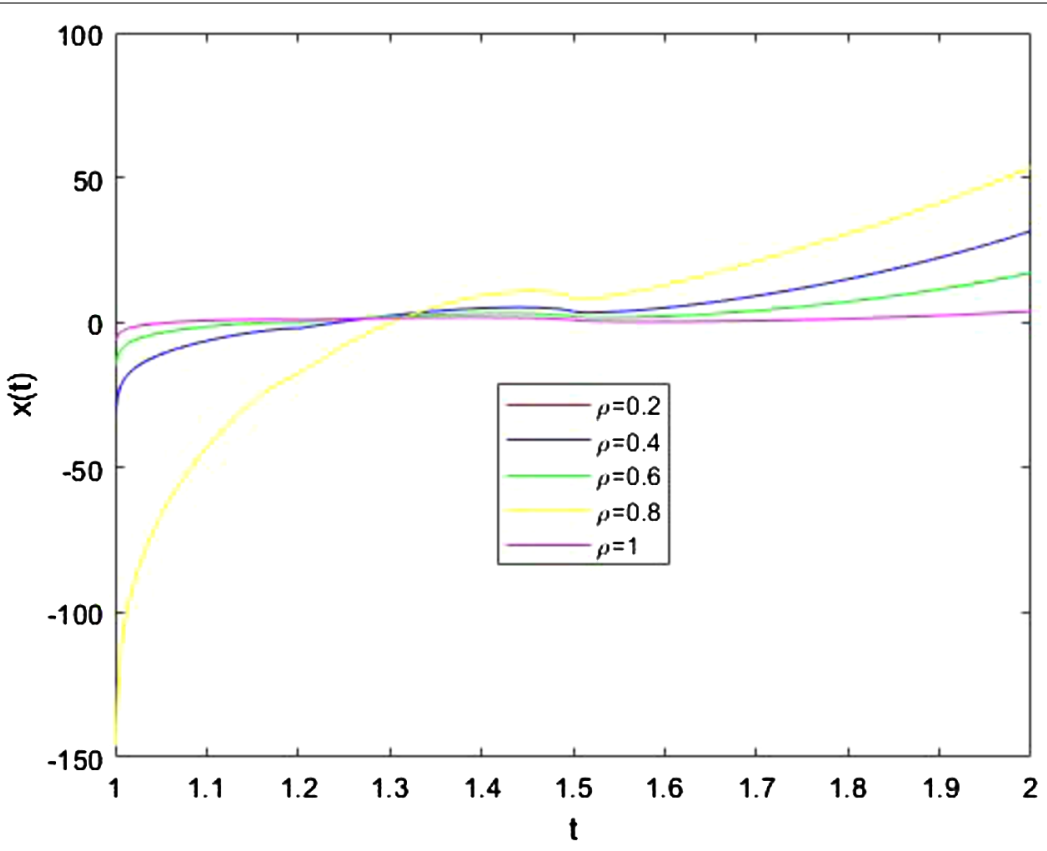

Figure 1 Graph of $x(t)$, for the Hilfer fractional derivatives $(\rho=1)$ and Hilfer generalized proportional fractional derivatives $(\rho \in(0,1))$

So, in view of (3.13) and (3.16), we have

$$
\mathcal{I}_{a^{+}}^{1-\gamma, \rho} x\left(a^{+}\right)=\sum_{i=1}^{m} c_{i} x\left(\tau_{i}\right)
$$

Hence, the proof is completed.

Remark 3.11 As shown below, the proposed Hilfer generalized proportional fractional derivative (see Definition 3.1) unifies the existing ones of Riemann-Liouville, the generalized proportional and the Hilfer fractional derivatives. We present the approximate numerical solution of Eq. (3.4) and present these solutions in Figs. 1-3.

\subsection{Uniqueness result}

This subsection will a detailed proof of the uniqueness of solutions of the proposed problem (1.1) using the concepts of the Banach contraction principle. Thus, we need the following assumptions.

$\left(H_{1}\right)$ Let $f: J \times \mathbb{R} \rightarrow \mathbb{R}$ be a function such that $f \in \mathcal{C}_{1-\gamma}^{q(1-p)}[J, \mathbb{R}]$ for any $x \in \mathcal{C}_{1-\gamma}^{\gamma}[J, \mathbb{R}]$.

$\left(H_{2}\right)$ There exists a constant $K>0$ such that

$$
|f(t, u)-f(t, \bar{u})| \leq K|u-\bar{u}|
$$

for any $u, \bar{u} \in \mathbb{R}$ and $t \in J$.

$\left(H_{3}\right)$ Suppose that

$$
K \psi<1,
$$




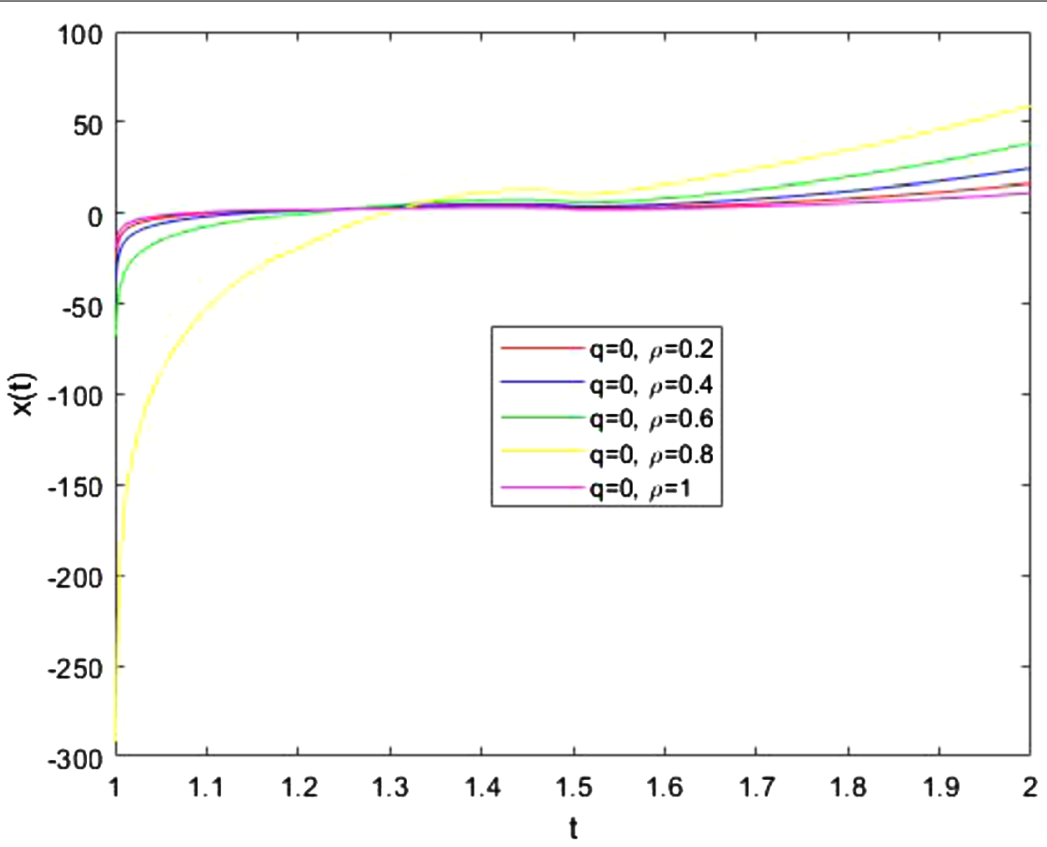

Figure 2 Graph of $x(t)$, for the Riemann-Liouville fractional derivatives $(q=0, \rho=1)$, and generalized proportional fractional derivatives $(q=0, \rho \in(0,1))$

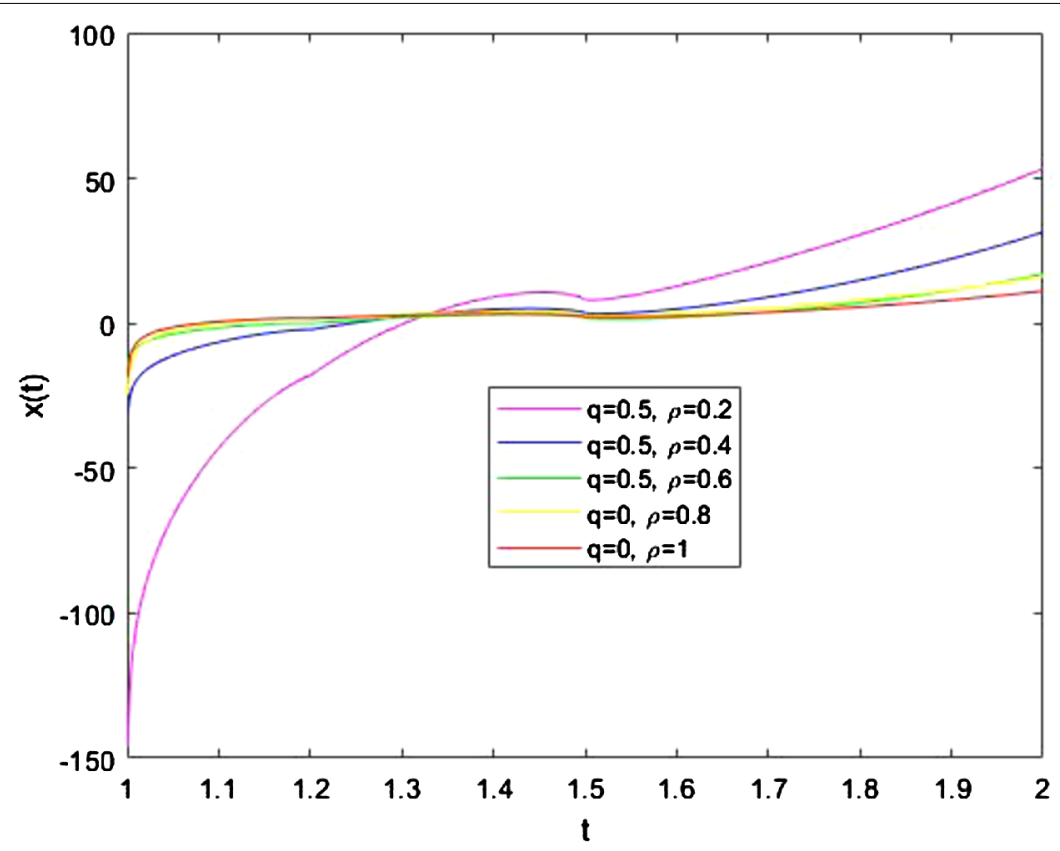

Figure 3 Graph of $x(t)$, for the Riemann-Liouville fractional derivatives $(q=0, \rho=1)$, generalized proportional fractional derivatives $(q=0, \rho=0.8)$ and Hilfer generalized proportional fractional derivatives $(q \in(0,1)$, $\rho \in(0,1))$

where

$$
\psi=\frac{\mathcal{B}(\gamma, p)}{\rho^{p} \Gamma(p)}\left(|\Lambda| \sum_{i=1}^{m} c_{i}\left(\tau_{i}-a\right)^{p+\gamma-1}+(T-a)^{p}\right)
$$


and $\mathcal{B}(\gamma, p)$ is the Beta function defined by [32]

$$
\mathcal{B}(\gamma, p)=\int_{0}^{1} x^{\gamma-1}(1-x)^{p-1} d x, \quad \operatorname{Re}(\gamma), \operatorname{Re}(p)>0
$$

Theorem 3.12 Let $0<p<1,0 \leq q \leq 1$ and $\gamma=p+q-p q$. Suppose that the assumptions $\left(H_{1}\right)-\left(H_{3}\right)$ are satisfied. Then problem $(1.1)$ has a unique solution in the space $\mathcal{C}_{1-\gamma}^{\gamma}[J, \mathbb{R}]$.

Proof Define the operator $T: \mathcal{C}_{1-\gamma}[J, \mathbb{R}] \rightarrow \mathcal{C}_{1-\gamma}[J, \mathbb{R}]$ by

$$
\begin{aligned}
(T x)(t)= & \frac{\Lambda}{\rho^{p} \Gamma(p)} e^{\frac{(\rho-1)}{\rho}(t-a)}(t-a)^{\gamma-1} \sum_{i=1}^{m} c_{i} \int_{a}^{\tau_{i}} e^{\frac{(\rho-1)}{\rho}\left(\tau_{i}-s\right)}\left(\tau_{i}-s\right)^{p-1} f(s, x(s)) d s \\
& +\frac{1}{\rho^{p} \Gamma(p)} \int_{a}^{t} e^{\frac{(\rho-1)}{\rho}(t-s)}(t-s)^{p-1} f(s, x(s)) d s .
\end{aligned}
$$

It follows that the operator $T$ is well defined. Now for any $x_{1}, x_{2} \in \mathcal{C}_{1-\gamma}[J, \mathbb{R}]$ and $t \in J$, this gives

$$
\begin{aligned}
& \left|\left(\left(T x_{1}\right)(t)-\left(T x_{2}\right)(t)\right)(t-a)^{1-\gamma}\right| \\
& \leq \frac{|\Lambda|}{\rho^{p} \Gamma(p)}\left|e^{\frac{(\rho-1)}{\rho}(t-a)}\right| \sum_{i=1}^{m} b_{i} \int_{a}^{\tau_{i}}\left|e^{\frac{(\rho-1)}{\rho}\left(\tau_{i}-s\right)}\right|\left(\tau_{i}-s\right)^{p-1}\left|f\left(s, x_{1}(s)\right)-f\left(s, x_{2}(s)\right)\right| d s \\
& \quad+\frac{1}{\rho^{p} \Gamma(p)} \int_{a}^{t}\left|e^{\frac{(\rho-1)}{\rho}(t-s)}\right|(t-s)^{p-1}\left|f\left(s, x_{1}(s)\right)-f\left(s, x_{2}(s)\right)\right| d s .
\end{aligned}
$$

Since $\left|e^{\frac{(\rho-1)}{\rho} t}\right|<1$, we get

$$
\begin{aligned}
\left|\left(\left(T x_{1}\right)(t)-\left(T x_{2}\right)(t)\right)(t-a)^{1-\gamma}\right| \\
\leq \frac{K|\Lambda|}{\rho^{p} \Gamma(p)}\left(\sum_{i=1}^{m} b_{i} \int_{a^{+}}^{\tau_{i}}\left(\tau_{i}-s\right)^{p-1}(s-a)^{\gamma-1} d s\right)\left\|x_{1}-x_{2}\right\|_{\mathcal{C}_{1-\gamma}[, \mathbb{R}]} \\
\quad+\frac{K}{\rho^{p} \Gamma(p)}(t-a)^{1-\gamma}\left(\int_{a^{+}}^{t}(t-s)^{p-1}(s-a)^{\gamma-1} d s\right)\left\|x_{1}-x_{2}\right\|_{\mathcal{C}_{1-\gamma}[, \mathbb{R}]} \\
\leq \frac{K|\Lambda|}{\rho^{p} \Gamma(p)} \mathcal{B}(\gamma, p) \sum_{i=1}^{m} c_{i}\left(\tau_{i}-a\right)^{p+\gamma-1}\left\|x_{1}-x_{2}\right\|_{\left.\mathcal{C}_{1-\gamma} U, \mathbb{R}\right]} \\
\quad+\frac{K}{\rho^{p} \Gamma(p)}(T-a)^{p} \mathcal{B}(\gamma, p)\left\|x_{1}-x_{2}\right\|_{\mathcal{C}_{1-\gamma}[, \mathbb{R}]} .
\end{aligned}
$$

Therefore,

$$
\begin{aligned}
& \left\|\left(T x_{1}\right)-\left(T x_{2}\right)\right\|_{\left.\mathcal{C}_{1-\gamma} J, \mathbb{R}\right]} \\
& \quad \leq \frac{K}{\rho^{p} \Gamma(p)} \mathcal{B}(\gamma, p)\left(|\Lambda| \sum_{i=1}^{m} c_{i}\left(\tau_{i}-a\right)^{p+\gamma-1}+(T-a)^{p}\right)\left\|x_{1}-x_{2}\right\|_{\mathcal{C}_{1-\gamma}[, \mathbb{R}]} \\
& \quad \leq K \psi\left\|x_{1}-x_{2}\right\|_{\left.\mathcal{C}_{1-\gamma} U, \mathbb{R}\right]} .
\end{aligned}
$$


Hence, it follows from (3.18) that $T$ is a contraction map. Thus, as a consequence of the Banach contraction principle, problem (1.1) has a unique solution.

\subsection{Existence result}

In this subsection, we prove the existence of solutions of problem (1.1) using the concepts of Kransnoselskii's fixed point theorem [33].

$\left(H_{4}\right)$ Suppose that

$$
K \Delta<1
$$

where

$$
\Delta=\frac{\mathcal{B}(\gamma, p)}{\rho^{p} \Gamma(p)}|\Lambda| \sum_{i=1}^{m} c_{i}\left(\tau_{i}-a\right)^{p+\gamma-1} .
$$

Theorem 3.13 Let $0<p<1,0 \leq q \leq 1$ and $\gamma=p+q-p q$. Suppose that the hypotheses $\left(H_{1}\right),\left(H_{2}\right)$ and $\left(H_{4}\right)$ are satisfied. Then problem (1.1) has at least one solution in the space $\mathcal{C}_{1-\gamma}^{\gamma}[J, \mathbb{R}]$.

Proof We have $\|\eta\|_{\mathcal{C}_{1-\gamma}[J, \mathbb{R}]}=\sup _{t \in J}\left|(t-a)^{1-\gamma} \eta(t)\right|$ and choose $\kappa \geq M\|\eta\|_{\mathcal{C}_{1-\gamma}[J, \mathbb{R}]}$, where

$$
M=\frac{\mathcal{B}(\gamma, p)}{\rho^{p} \Gamma(p)}\left(|\Lambda| \sum_{i=1}^{m} c_{i}\left(\tau_{i}-a\right)^{p+\gamma-1}+(T-a)^{p}\right),
$$

we consider $\mathbf{B}_{\kappa}=\left\{x \in \mathbb{C}[J, \mathbb{R}]:\|x\|_{\mathcal{C}_{1-\gamma}[J, \mathbb{R}]} \leq \kappa\right\}$. Define the operators $\mathbb{T}_{1}$ and $\mathbb{T}_{2}$ on $\mathbf{B}_{\kappa}$ by

$$
\begin{aligned}
& \mathbb{T}_{1} x(t)=\frac{1}{\rho^{p} \Gamma(p)} \int_{a}^{t} e^{\frac{(\rho-1)}{\rho}(t-s)}(t-s)^{p-1} f(s, x(s)) d s, \\
& \mathbb{T}_{2} x(t)=\frac{\Lambda}{\rho^{p} \Gamma(p)} e^{\frac{(\rho-1)}{\rho}(t-a)}(t-a)^{\gamma-1} \sum_{i=1}^{m} c_{i} \int_{a}^{\tau_{i}} e^{\frac{(\rho-1)}{\rho}\left(\tau_{i}-s\right)}\left(\tau_{i}-s\right)^{p-1} f(s, x(s)) d s,
\end{aligned}
$$

for all $t \in[a, T]$. Now, for every $x, y \in \mathbf{B}_{\kappa}$,

$$
\begin{aligned}
& \left|\left(\mathbb{T}_{1} x(t)+\mathbb{T}_{2} y(t)\right)(t-a)^{1-\gamma}\right| \\
& \leq \frac{(t-a)^{1-\gamma}}{\rho^{p} \Gamma(p)} \int_{a}^{t}(t-s)^{p-1}(s-a)^{\gamma-1}\left|f(s, x(s))(s-a)^{1-\gamma}\right| d s \\
& \quad+\frac{|\Lambda|}{\rho^{p} \Gamma(p)} \sum_{i=1}^{m} c_{i} \int_{a}^{\tau_{i}}\left(\tau_{i}-s\right)^{p-1}\left(\tau_{i}-a\right)^{\gamma-1}\left|f(s, y(s))\left(\tau_{i}-a\right)^{1-\gamma}\right| d s \\
& \leq\|\eta\|\left[\frac{\mathcal{B}(\gamma, p)}{\rho^{p} \Gamma(p)}|\Lambda| \sum_{i=1}^{m} c_{i}\left(\tau_{i}-a\right)^{p+\gamma-1}+\frac{\mathcal{B}(\gamma, p)}{\rho^{p} \Gamma(p)}(T-a)^{p}\right] \\
& \leq\|\eta\| M \\
& \leq \kappa<\infty .
\end{aligned}
$$

This implies that $\mathbb{T}_{1} x+\mathbb{T}_{2} y \in \mathbf{B}_{\kappa}$. 
Step 2. We show that $\mathbb{T}_{2}$ is a contraction.

Now, let $x, y \in \mathcal{C}_{1-\gamma}[J, \mathbb{R}]$ and $t \in J$, then

$$
\begin{aligned}
& \left|\left(\mathbb{T}_{2} x(t)-\mathbb{T}_{2} y(t)\right)(t-a)^{1-\gamma}\right| \\
& \quad=\left|\Lambda e^{\frac{(\rho-1)}{\rho}(t-a)} \sum_{i=1}^{m} c_{i} \mathcal{I}_{a^{+}}^{p, \rho}(f(s, x(s))-f(s, y(s)))\left(\tau_{i}\right)\right| \\
& \quad \leq \frac{K|\Lambda|}{\rho^{p} \Gamma(p)} \sum_{i=1}^{m} c_{i} \int_{a}^{\tau_{i}}\left(\tau_{i}-s\right)^{p-1}\left(\tau_{i}-s\right)^{\gamma-1}|x(s)-y(s)| d s \\
& \quad \leq\left[\frac{K|\Lambda|}{\rho^{p} \Gamma(p)} \mathcal{B}(\gamma, p) \sum_{i=1}^{m} c_{i}\left(\tau_{i}-a\right)^{p+\gamma-1}\right]\|x-y\|_{\mathcal{C}_{1-\gamma}[, \mathbb{R}]} \\
& \quad \leq K \Delta\|x-y\|_{\mathcal{C}_{1-\gamma}[, \mathbb{R}]} .
\end{aligned}
$$

Hence, it follows from $\left(H_{4}\right)$ that $\mathbb{T}_{2}$ is a contraction.

Step 3. We show that the operator $\mathbb{T}_{1}$ is continuous and compact.

Clearly, the operator $\mathbb{T}_{1}$ is continuous, due to the fact that the function $f$ is continuous.

Thus, for any $x \in \mathcal{C}_{1-\gamma}[J, \mathbb{R}]$, we have

$$
\left\|\mathbb{T}_{1} x\right\| \leq\|\eta\| \frac{\mathcal{B}(\gamma, p)}{\rho^{p} \Gamma(p)}(T-a)^{p}<\infty .
$$

This shows that the operator $\mathbb{T}_{1}$ is uniformly bounded on $\mathbf{B}_{\kappa}$. Thus, it remains to shows that $\mathbb{T}_{1}$ is compact. Denoting $\sup _{(t, x) \in J \times \mathbf{B}_{\kappa}}|f(t, x(t))|=\delta<\infty$ and for any $a<\tau_{1}<\tau_{2}<T$,

$$
\begin{aligned}
\left|\left(\tau_{2}-a\right)^{1-\gamma}\left(\mathbb{T}_{1} x\left(\tau_{2}\right)\right)+\left(\tau_{1}-a\right)^{1-\gamma}\left(\mathbb{T}_{1} x\left(\tau_{1}\right)\right)\right| \\
=\mid \frac{\left(\tau_{2}-a\right)^{1-\gamma}}{\rho^{p} \Gamma(p)} \int_{a}^{\tau_{2}} e^{\frac{(\rho-1)}{\rho}\left(\tau_{2}-s\right)}\left(\tau_{2}-s\right)^{p-1} f(s, x(s)) d s \\
\quad-\frac{\left(\tau_{1}-a\right)^{1-\gamma}}{\rho^{p} \Gamma(p)} \int_{a}^{\tau_{1}} e^{\frac{(\rho-1)}{\rho}\left(\tau_{1}-s\right)}\left(\tau_{1}-s\right)^{p-1} f(s, x(s)) d s \mid \\
\leq \frac{1}{\rho^{p} \Gamma(p)} \int_{a}^{\tau_{2}}\left[\left(\tau_{2}-a\right)^{1-\gamma}\left(\tau_{2}-s\right)^{p-1}-\left(\tau_{1}-a\right)^{1-\gamma}\left(\tau_{1}-s\right)^{p-1}\right]|f(s, x(s))| d s \\
\quad+\frac{1}{\rho^{p} \Gamma(p)} \int_{\tau_{1}}^{\tau_{2}}\left(\tau_{2}-a\right)^{1-\gamma}\left(\tau_{2}-s\right)^{p-1}|f(s, x(s))| d s \\
\rightarrow 0, \quad \text { as } \tau_{2} \rightarrow \tau_{1} .
\end{aligned}
$$

As a consequences of Arzelá-Ascoli theorem, the operator $\mathbb{T}_{1}$ is compact on $\mathbf{B}_{\kappa}$. Thus, problem (1.1) has at least one solution.

\subsection{Examples}

Example 3.14 Consider the fractional differential equation which involves the Hilfer generalized proportional derivative of the form

$$
\left\{\begin{array}{l}
\mathcal{D}_{0^{+}}^{\frac{2}{3}, \frac{1}{2}, 1} x(t)=\frac{1}{25 e^{2 t}}\left(\frac{\cos 2 t}{1+|x(t)|}\right)+\frac{3}{2}, \quad t \in J=[0,2], \\
\mathcal{I}_{0^{+}}^{1-\gamma, 1} x(0)=2 x\left(\frac{2}{5}\right)
\end{array}\right.
$$


By comparing (1.1) with (3.28), we get $p=\frac{2}{3}, q=\frac{1}{2}, \rho=1, \gamma=\frac{5}{6}, a=0, T=2, c_{1}=2$ since $m=1, \tau_{1}=\frac{2}{5} \in J$ and $f: J \times \mathbb{R} \rightarrow \mathbb{R}$ is a function defined by

$$
f(t, u)=\frac{1}{25 e^{2 t}}\left(\frac{\cos 2 t}{1+|u|}\right)+\frac{3}{2}, \quad t \in J, u \in \mathbb{R}_{+} .
$$

Thus, $f$ is continuous and for all $u, v \in \mathbb{R}_{+}$and $t \in J$, we have $|f(t, u)-f(t, v)| \leq \frac{1}{25}|u-v|$. Thus, it follows that conditions $\left(H_{1}\right)$ and $\left(H_{3}\right)$ are true with $K=\frac{1}{25}$. Therefore, by simple calculation, we can see that $|\Lambda| \approx 0.8325$ and $\psi \approx 3.3628$, which implies that

$$
K \psi \approx 0.1345<1
$$

Hence, all the assumptions of Theorem 3.12 are satisfied. So, problem (1.1) has a unique solution on $J$.

Similarly, we find that $\Delta \approx 1.3413>0$ and $K \Delta \approx 0.0537<1$. Since all the hypotheses of Theorem 3.13 hold, we conclude that problem (1.1) has at least one solution on $J$.

Example 3.15 Consider the Hilfer generalized proportional fractional differential equation described by

$$
\left\{\begin{array}{l}
\mathcal{D}_{0^{+}}^{\frac{2}{3}, \frac{1}{2}, \frac{1}{5}} x(t)=\frac{1}{25 e^{2 t}}\left(\frac{\cos 2 t}{1+|x(t)|}\right)+\frac{3}{2}, \quad t \in J=[0,2] \\
\mathcal{I}_{0^{+}}^{1-\gamma, \frac{1}{5}} x(0)=2 x\left(\frac{2}{5}\right) .
\end{array}\right.
$$

Repeating application of the same procedure as Example 3.14 above, we get the values $|\Lambda| \approx 0.9943, \psi \approx 10.5950$ and $\Delta \approx 4.6839$. Thus

$$
K \psi \approx 0.4238<1
$$

According to Theorem 3.12, problem (1.1) has a unique solution on $J$. In addition,

$$
K \Delta \approx 0.1874<1
$$

hence, by Theorem 3.13, problem (1.1) has at least one solution on $J$.

It should be noted here that the proposed Hilfer generalized proportional derivative (3.1) unifies the existing ones in the sense of Riemann-Liouville and Caputo generalized proportional fractional derivative, respectively. In addition:

- If $\rho \rightarrow 1$ and $q \in[0,1]$, the formulation for this problem, reduce to Hilfer fractional derivative [18, 32, 44] (see Fig. 1).

- If $\rho \in(0,1)$ and $q \in[0,1]$, we obtain the proposed Hilfer generalized proportional fractional derivative, which we can see that it covers the classical Hilfer fractional derivative, as shown in Fig. 1.

- If $\rho \rightarrow 1$ and $q=0$, the formulation for this problem reduces to the Riemann-Liouville fractional derivative [32] (see Fig. 2).

- If $\rho \rightarrow 1$ and $q=1$, we obtain the Caputo fractional derivative [32]. 
- If $\rho \in(0,1)$ and $q=0$, we obtain the Riemann-Liouville generalized proportional fractional derivative [23], which we can see from Fig. 2 to cover the Riemann-Liouville fractional derivative.

- If $\rho \in(0,1)$ and $q=1$, we obtain the Caputo generalized proportional fractional derivative [23].

- If $q, \rho \in(0,1)$, it is easily to observe from Fig. 3 that the newly proposed derivative unifies the ones in the setting of the Hilfer, Riemann-Liouville and generalized proportional fractional derivatives.

\title{
4 Conclusions
}

In this paper, we defined the proportional fractional derivatives in the Hilfer setting. We used some known theorems from the fixed point theory that enabled us to prove the existence and uniqueness of solutions to a specific type of fractional initial value problem involving the Hilfer proportional fractional derivative. Furthermore, to show the effectiveness of our results, we presented some examples. In fact, the Hilfer proportional derivative contains three parameters. The existence of more parameters is useful especially when one considers the stability and other qualitative aspects of differential equations involving fractional derivative.

\begin{abstract}
Acknowledgements
The authors acknowledge the financial support provided by the Center of Excellence in Theoretical and Computational Science (TaCS-CoE), KMUTT. The first and last authors were supported by the "Petchra Pra Jom Klao Ph.D. Research Scholarship from King Mongkut's University of Technology Thonburi". (For Ph.D. Petchra Pra Jom Klao Doctoral Scholarship) (Grant No. 13/2561). Moreover, this research work was financially supported by King Mongkut's University of Technology Thonburi through the KMUTT 55th Anniversary Commemorative Fund.
\end{abstract}

Funding

Petchra Pra Jom Klao Doctoral Scholarship for Ph.D. program of King Mongkut's University of Technology Thonburi (KMUTT). The Center of Excellence in Theoretical and Computational Science (TaCS-CoE), KMUTT.

Availability of data and materials

Data sharing is not applicable to this paper, as no data set was generated or analyzed during the current study.

\section{Competing interests}

The authors declare no conflict of interest.

\section{Authors' contributions}

In writing this article the authors have contributed equally. Both authors read and approved the final manuscript.

\section{Author details}

${ }^{1}$ KMUTTFixed Point Research Laboratory, KMUTT-Fixed Point Theory and Applications Research Group (KMUTT-FPTA), Department of Mathematics, Room SCL 802 Fixed Point Laboratory, Science Laboratory Building, Faculty of Science, King Mongkut's University of Technology Thonburi (KMUTT), 126 Pracha-Uthit Road, Bang Mod, Thrung Khru, Bangkok 10140, Thailand. '2Department of Medical Research, China Medical University Hospital, China Medical University, Taichung 40402, Taiwan. ${ }^{3}$ Department of Mathematics and Computer Science, Sule Lamido University, P. M. B 048 Kafin-Hausa, Jigawa State, Nigeria. ${ }^{4}$ Department of Mathematics, Çankaya University, Ankara, 06790, Turkey.

\section{Publisher's Note}

Springer Nature remains neutral with regard to jurisdictional claims in published maps and institutional affiliations.

Received: 15 May 2020 Accepted: 22 June 2020 Published online: 02 July 2020

References

1. Abdeljawad, T.: On conformable fractional calculus. J. Comput. Appl. Math. 279, 57-66 (2015)

2. Ahmed, I., Kumam, P., Jarad, F., Borisut, P., Sitthithakerngkiet, K., Ibrahim, A.: Stability analysis for boundary value problems with generalized nonlocal condition via Hilfer-Katugampola fractional derivative. Adv. Differ. Equ. 2020(1), 225 (2020)

3. Ahmed, I., Kumam, P., Shah, K., Borisut, P., Sitthithakerngkiet, K., Demba, M.A.: Stability results for implicit fractional pantograph differential equations via $\phi$-Hilfer fractional derivative with a nonlocal Riemann-Liouville fractional integral condition. Mathematics 8(1), 94 (2020) 
4. Almeida, R.: A Caputo fractional derivative of a function with respect to another function. Commun. Nonlinear Sci. Numer. Simul. 44, 460-481 (2017)

5. Anatoly, A.K.: Hadamard-type fractional calculus. J. Korean Math. Soc. 38(6), 1191-1204 (2001)

6. Anderson, D.: Second-order self-adjoint differential equations using a proportional-derivative controller. Commun. Appl. Nonlinear Anal. 24(1), 17-48 (2017)

7. Anderson, D.R., Ulness, D.J.: Newly defined conformable derivatives. Adv. Dyn. Syst. Appl. 10(2), 109-137 (2015)

8. Asawasamrit, S., Kijjathanakorn, A., Ntouyas, S.K., Tariboon, J.: Nonlocal boundary value problems for Hilfer fractional differential equations. Bull. Korean Math. Soc. 55(6), 1639-1657 (2018)

9. Atangana, A.: Derivative with a New Parameter: Theory, Methods and Applications. Academic Press, San Diego (2015)

10. Atangana, A.: Fractional Operators with Constant and Variable Order with Application to Geo-Hydrology. Academic Press, San Diego (2017)

11. Atangana, A., Baleanu, D.: Application of fixed point theorem for stability analysis of a nonlinear Schrodinger with Caputo-Liouville derivative. Filomat 31(8), 2243-2248 (2017)

12. Atangana, A., Baleanu, D., Alsaedi, A.: New properties of conformable derivative. Open Math. 13, $889-898$ (2015)

13. Atangana, A., Goufo, E.F.D.: Cauchy problems with fractal-fractional operators and applications to groundwater dynamics. Fractals (2020, in press). https://doi.org/10.1142/S0218348X20400435

14. Atangana, A., Koca, I.: New direction in fractional differentiation. Math. Nat. Sci. 1, 18-25 (2017)

15. Atangana, A., Secer, A.: A note on fractional order derivatives and table of fractional derivatives of some special functions. In: Abstract and Applied Analysis, vol. 2013. Hindawi (2013)

16. Borisut, P., Kumam, P., Ahmed, I., Sitthithakerngkiet, K.: Nonlinear Caputo fractional derivative with nonlocal Riemann-Liouville fractional integral condition via fixed point theorems. Symmetry 11(6), 829 (2019)

17. Debnath, L.: Recent applications of fractional calculus to science and engineering. Int. J. Math. Math. Sci., 54, 3413-3442 (2003)

18. Furati, K.M., Kassim, M.D., et al.: Existence and uniqueness for a problem involving Hilfer fractional derivative. Comput. Math. Appl. 64(6), 1616-1626 (2012)

19. Gambo, Y.Y., Jarad, F., Baleanu, D., Abdeljawad, T.: On Caputo modification of the Hadamard fractional derivatives. Adv. Differ. Equ. 2014, 10 (2014)

20. Harikrishnan, S., Shah, K., Baleanu, D., Kanagarajan, K.: Note on the solution of random differential equations via $\psi$-Hilfer fractional derivative. Adv. Differ. Equ., 2018, 224 (2018)

21. Hilfer, R., et al.: Applications of Fractional Calculus in Physics, vol. 35. World Scientific, Singapore (2000)

22. Jarad, F., Abdeljawad, T.: Generalized fractional derivatives and Laplace transform. Discrete Contin. Dyn. Syst., Ser. S 13(3), 709-722 (2020)

23. Jarad, F., Abdeljawad, T., Alzabut, J.: Generalized fractional derivatives generated by a class of local proportional derivatives. Eur. Phys. J. Spec. Top. 226(16-18), 3457-3471 (2017)

24. Jarad, F., Abdeljawad, T., Baleanu, D.: Caputo-type modification of the Hadamard fractional derivatives. Adv. Differ. Equ. 2012(1), 142 (2012)

25. Jarad, F., Abdeljawad, T., Baleanu, D.: On the generalized fractional derivatives and their Caputo modification. J. Nonlinear Sci. Appl. 10(5), 2607-2619 (2017)

26. Jarad, F., Harikrishnan, S., Shah, K., Kanagarajan, K.: Existence and stability results to a class of fractional random implicit differential equations involving a generalized Hilfer fractional derivative. Discrete Contin. Dyn. Syst., Ser. S 13(3), $723(2020)$

27. Jarad, F., Uğurlu, E., Abdeljawad, T., Baleanu, D.: On a new class of fractional operators. Adv. Differ. Equ. 2017, 247 (2017)

28. Katugampola, U.N.: New approach to a generalized fractional integral. Appl. Math. Comput. 218(3), 860-865 (2011)

29. Katugampola, U.N.: A new approach to generalized fractional derivatives. Bull. Math. Anal. Appl. 6(4), 1-15 (2014)

30. Khalil, R., Al Horani, M., Yousef, A., Sababheh, M.: A new definition of fractional derivative. J. Comput. Appl. Math. 264, 65-70 (2014)

31. Khan, M.A., Atangana, A.: Modeling the dynamics of novel coronavirus (2019-ncov) with fractional derivative. Alex Eng. J. (2020, in press). https://doi.org/10.1016/j.aej.2020.02.033

32. Kilbas, A., Srivastava, H., Trujillo, J.: Theory and Applications of Fractional Derivatial Equations. North-Holland Mathematics Studies, vol. 204. North-Holland, Amsterdam (2006)

33. Krasnoselskii, M.: Two remarks about the method of successive approximations. Usp. Mat. Nauk 10, $123-127$ (1955)

34. Magin, R.L.: Fractional Calculus in Bioengineering, vol. 2. Begell House, Redding (2006)

35. Mainardi, F.: Fractional Calculus and Waves in Linear Viscoelasticity: An Introduction to Mathematical Models. World Scientific, Singapore (2010)

36. Oliveira, D de Oliveira, E. C. Hilfer-Katugampola fractional derivatives. Comput. Appl. Math. 37(3), 3672-3690 (2018)

37. Osler, T.J.: The fractional derivative of a composite function. SIAM J. Math. Anal. 1(2), $288-293$ (1970)

38. Owolabi, K.M., Atangana, A., Akgul, A.: Modelling and analysis of fractal-fractional partial differential equations: application to reaction-diffusion model. Alex. Eng. J. (2020, in press). https://doi.org/10.1016/j.aej.2020.03.022

39. Podlubny, I.: Fractional Differential Equations: An Introduction to Fractional Derivatives, Fractional Differential Equations, to Methods of Their Solution and Some of Their Applications, vol. 198. Elsevier, Amsterdam (1998)

40. Riaz, M.B., Atangana, A., Abdeljawad, T.: Local and nonlocal differential operators: a comparative study of heat and mass transfer in mhd oldroyd-b fluid with ramped wall temperature. Fractals (2020, in press). https://doi.org/10.1142/S0218348X20400332

41. Samko, S.G., Kilbas, A.A., Marichev, O.I., et al.: Fractional Integrals and Derivatives, vol. 1993. Gordon \& Breach, Yverdon (1993)

42. Shah, K., Vivek, D., Kanagarajan, K.: Dynamics and stability of $\psi$-fractional pantograph equations with boundary conditions. Bol. Soc. Parana. Mat. 22(2), 1-13 (2018)

43. Shammakh, W., Alzumi, H.Z.: Existence results for nonlinear fractional boundary value problem involving generalized proportional derivative. Adv. Differ. Equ. 2019, 94 (2019)

44. Vivek, D., Kanagarajan, K., Elsayed, E.: Some existence and stability results for Hilfer-fractional implicit differential equations with nonlocal conditions. Mediterr. J. Math. 15(1), 15 (2018)

45. Vivek, D., Shah, K., Kanagarajan, K.: Existence theory and continuation analysis of nonlinear pantograph equations via Hilfer-Hadamard fractional derivative. Dyn. Contin. Discrete Impuls. Syst., Ser. A Math. Anal. 25, 397-417 (2018) 